\title{
$D$-REGULARITY
}

\section{NATHAN DIVINSKY}

We shall call an element $x$ of a ring $A$, right $D$-regular if there exists an element $y$ in $A$ such that $x=x y$. This property of $x$ belonging to $x A$ has been studied before $[2 ; 6] .{ }^{1}$ With techniques available from [5] it is not difficult to show the existence of a maximal right $D$ regular two-sided ideal $M_{R}$ and a left analogue $M_{L}$. These are in general not equal. They are connected with the Jacobson radical $J$ and the subradicals $P_{R}$ and $P_{L}$, [6], in the following way:

$$
P_{R}=J \cap M_{R} ; \quad P_{L}=J \cap M_{L} .
$$

The present note goes on to consider the cases $M_{R}=0$ and $M_{R}=A$, for various degrees of chain assumptions. In the commutative case $M_{R}=M_{L}=M$, the maximal $D$-regular ideal.

1. Preliminaries. Following Brown and McCoy [5], to each element $a$ of a ring $A$ we associate the right ideal $F(a)=a A$. The element $a$ is said to be right $D$-regular (r.D.r.) if $a$ belongs to $F(a)$. A right ideal is said to be r.D.r. if every element in it is r.D.r. It is easy to see that $F(a+b) \leqq F(a)+(b)_{r} \leqq F(a)+(b)$, and that $F(a+b) \leqq F(a)$ if $b$ is in $F(a)$. Then by Theorems 1 and 2 of [5] we can conclude that $M_{R}$ $=\{x:(x)$ is r.D.r. $\}$, is a two-sided ideal which contains every r.D.r. two-sided ideal; and that $M_{R}\left(A-M_{R}\right)=0$. In other words, $A$ is an $\left(F, \Omega, \Omega^{\prime}\right)$ group and by Theorem 6 of $[5]$ we have:

$$
M_{R}=\bigcap_{i} M_{i}^{\prime}
$$

where $M_{2}$ is a large modular right ideal, i.e. there exists an element $x_{i}$ not in $M_{i}$ such that $x_{i} A \leqq M_{i}$ and such that every right ideal which properly contains $M_{i}$, also contains $x_{i}$. The set $M_{i}^{\prime}$ is the largest twosided ideal contained in $M_{i}$.

Though this development is both elegant and general it does not seem to yield the fact that $M_{R}$ contains all the r.D.r. right ideals. In particular, if $x$ is in $x A$ and if for every $y$ of $A, x y$ is in $x y A$, it is not clear that $x$ must be in $M_{R}$. To obtain this fact one must return to the original Jacobson techniques and develop $M_{R}$ from a one sided point of view. Using a technique of [6] we obtain:

Presented to the Society, August 23, 1956; received by the editors November 25. 1955 and, in revised form, May 13, 1957.

1 Numbers in square brackets refer to the bibliography at the end of the paper. 
Lemma 1. If $x$ is r.D.r. and if a belongs to an r.D.r. right ideal, namely $a A$, then $a+x$ is r.D.r.

Proof. We have $x x^{\prime}=x, a a^{\prime}=a$. Define $u=a-a x^{\prime}=a a^{\prime}-a x^{\prime}$ $=a\left(a^{\prime}-x^{\prime}\right)$. Then $u$ belongs to $a A$ and therefore there exists an element $u^{\prime}$ such that $u u^{\prime}=u$. Define $v=u^{\prime}+x^{\prime}-x^{\prime} u^{\prime}$. Then $x v=x u^{\prime}$ $+x x^{\prime}-x x^{\prime} u^{\prime}=x u^{\prime}+x-x u^{\prime}=x$; and $a v=a u^{\prime}+a x^{\prime}-a x^{\prime} u^{\prime}=\left(a-a x^{\prime}\right) u^{\prime}$ $+a x^{\prime}=a-a x^{\prime}+a x^{\prime}=a$. Therefore $(a+x) v=a+x$. Q.E.D.

COROllary. The sum or two r.D.r. right ideals is an r.D.r. right ideal.

We define $M_{R}^{*}$ to be the union of all the r.D.r. right ideals. Then $M_{R}^{*}$ is itself an r.D.r. right ideal. We now show that it is a two-sided ideal:

Lemma 2. $M_{R}^{*}$ is a two-sided ideal of $A$.

Proof. For $a$ in $A$ and $x$ in $M_{R}^{*}, x a$ is in $M_{R}^{*}$. Since $x x^{\prime}=x$ and for every $y$ of $A$, there is an element $y^{\prime}$ such that $x y=x y y^{\prime} ; a x x^{\prime}=a x$ and $a x y y^{\prime}=a x y$. Therefore $a x$ is in $M_{R}^{*}$. Q.E.D.

It is now easy to see that $M_{R}^{*}=M_{R}$ and we have

THEOREM 1. If $x$ is in $x A$ and for every $y$ of $A, x y$ is in $x y A$, then $x$ is in $M_{R}$.

The proof of the next theorem is immediate from results in [6].

Theorem 2. If $J$ is the Jacobson radical of $A$, then $P_{R}=J \cap M_{R}$ $=J\left(M_{R}\right) ;$ and $P_{L}=J \cap M_{L}=J\left(M_{L}\right)$.

Thus in the commutative case, the subradical is the radical of the max.D.r. ideal.

We shall now obtain some simple properties of $M_{R}$. First we observe that $M_{R}$ and $M_{L}$ may not be equal. The following example is due to Hopkins [8]. Let $A$ be the set of all $m e+n u$, where $e^{2}=e$, $u^{2}=0, e u=u, u e=0$, and where $m, n$ are in a field $F$. Then $M_{L}=A$ whereas $M_{R}=0$. The proof of the following lemma is immediate.

Lemma 3. If $B$ is an ideal of $A$, then $M_{R}(B)<B \cap M_{R}$.

The point is that they may not be equal. Let $B=J$, and let $A$ have a right unity element. Then $M_{R}(B)=0$, whereas $B \cap M_{R}=J \cap A=J$.

We observe also that $M_{R}=M_{R} A=M_{R} A^{n}$ for every $n ; M_{L}=A^{m} M_{L}$, for every $m$.

Lemma 4. $M_{R}\left(M_{R}\right) \leqq M_{R}^{n}$ for every $n ; M_{R}\left(M_{R}\right)=M_{R}\left(M_{R}^{n}\right)$ for every $n$.

Proof. If $x$ is in $M_{R}\left(M_{R}\right)$, then in particular $x=x y$, with $y$ in $M_{R}$. 
Then $x=x y^{n-1}$, which is in $M_{R}^{n}$. The second half is also immediate, $x=x y^{n}$, with $y^{n}$ in $M_{R}^{n}$.

Thus we have $M_{R}>M_{R}^{2}>\ldots>M_{R}^{n}>\ldots>M_{R}\left(M_{R}\right)$ $=M_{R}\left(M_{R}^{2}\right)=\cdots=M_{R}\left(M_{R}^{n}\right)=\cdots$.

This chain can in fact descend.

ExAmple 1. Let $A$ be the set of all finite sums $\sum_{1}^{n} \alpha_{i} x^{i}+\sum_{1}^{m} \beta_{j} y^{i}$, where $\alpha_{i}$ and $\beta_{j}$ are in a field $F$ and where $x$ and $y$ are indeterminates such that $x^{i} y^{j}=y^{j} x^{i}=x^{i}$ for every $i$ and $j$. Then $M=(x), M^{2}$ $=\left(x^{2}\right), \cdots, M^{n}=\left(x^{n}\right), M(M)=\cdots=M\left(M^{n}\right)=\cdots=0$.

By methods almost the same as in [4], one can prove

Theorem 3. If $A_{n}$ is the complete matric ring of order $n$ over $A$ then

$$
M_{R}\left(A_{n}\right)=\left(M_{R}(A)\right)_{n} ; \quad M_{L}\left(A_{n}\right)=\left(M_{L}(A)\right)_{n} .
$$

This leads to

Lemma 5. If $a_{1}, \cdots, a_{n}$ is any finite set of elements in $M_{R}, a_{i}=a_{i} a_{i}^{\prime}$, then there exists an element $b$ in $A$ but not necessarily in $M_{R}$ such that $a_{i}=a_{i} b$ for all the $a_{i}$.

Proof. Since the $a_{i}$ are in $M_{R}$, the $n \times n$ matrix $c$, with the $a_{i}$ in the first column and zeros elsewhere, is in $M_{R}\left(A_{n}\right)$, by Theorem 3 . Then, in particular, there exists a matrix $d=\left(b_{i j}\right)$ such that $c d=c$. However

$$
c d=\left(\begin{array}{c}
a_{1} b_{11} \cdots \\
a_{2} b_{11} \cdots \\
\vdots \\
a_{n} b_{11}
\end{array}\right)
$$

and therefore $b=b_{11}$.

An inductive proof can also be given. For $n=2$, Lemma 5 is a consequence of the proof of Lemma 1 . We assume the result for any set of $n-1$ elements of $M_{R}$. Given $a_{1}, \cdots, a_{n}$, with $a_{i}=a_{i} a_{i}^{\prime}$, consider the set of $n-1$ elements $a_{i}-a_{i} a_{n}{ }^{\prime}$, for $i=1, \cdots, n-1$. By induction there exists an element $g$ such that $\left(a_{i}-a_{i} a_{n}^{\prime}\right) g=a_{i}-a_{i} a_{n}^{\prime}$. Define $b=a_{n}^{\prime}+g-a_{n}^{\prime} g$. Then $a_{n} b=a_{n}+a_{n} g-a_{n} g=a_{n} ;$ whereas $a_{i} b=a_{i} a_{n}^{\prime}$ $+\left(a_{i}-a_{i} a_{n}^{\prime}\right) g=a_{i} a_{n}^{\prime}+a_{i}-a_{i} a_{n}^{\prime}=a_{i}$, for $i=1, \cdots, n-1$. Q.E.D.

COROLlary. If $M_{R}$ is finitely generated as a left $A$-module, then there exists an element $e$ in $A$ such that $M_{R}=M_{R}$ e pointwise. The element $e$ is not necessarily in $M_{R}$, or an idempotent or unique.

It is clear that if $A$ has a right unity element then $A=M_{R}$. If 
$A=J$, then both $M_{R}$ and $M_{L}$ are zero, since $x$ in $x J$ or $J x$ implies $x=0,[3]$. Thus we might expect that if $M_{R}=A$ then $A$ is well behaved whereas if $M_{R}=M_{L}=0$ then $A$ is radical-like. This is certainly so when $A$ is commutative with DCC. In that case $A$ can be expressed as $A=e A+N_{0}$ where $e$ is an idempotent, $e N_{0}=0$ and $N_{0}$ is nilpotent. Since a central idempotent is always in both $M_{R}$ and $M_{L}$, here $e$ is in $M$ and thus $e A \leqq M$. On the other hand if $x$ is in $M, x$ $=e x_{1}+n_{1}$, then in particular there exists an element $e y+n_{2}$ such that $\left(e x_{1}+n_{1}\right) \cdot\left(e y+n_{2}\right)=e x_{1}+n_{1}$. Then $e x_{1} y+n_{1} n_{2}=e x_{1}+n_{1}$ and thus $n_{1} n_{2}$ $=n_{1}, n_{1}=0$. Therefore $M \leqq e A, M=e A$. Also $N_{0}$ is simply $M^{\prime}$, the set of annihilators of $M$. We have

Theorem 4. If $A$ is a commutative ring with $D C C$ then $A=M+M^{\prime}$, where $M$, the max.D.r. ideal, has a unity element and $M^{\prime}$ is nilpotent.

This corresponds to the result in [4] which states that every ring $A$ with DCC (though not necessarily commutative) can be expressed as $\bar{M}+\bar{M}^{\prime}$ where $\bar{M}$, the max. regular ideal, is semi-simple, and where $\bar{M}^{\prime}$ is bound to its radical. Here $M>\bar{M}$ and $M^{\prime}<\bar{M}^{\prime}$. Thus we know more about $M^{\prime}$, namely that it is nilpotent, and less about $M$, since it is not necessarily semi-simple.

Corollary 1. If $A$ is commutative with DCC then $M=A$ if and only if $A$ has a unity element; $M=0$ if and only if $A$ is nilpotent.

Using the fact that $M(A-M)=0$ we then have

Corollary 2. If $A$ is commutative with DCC for $A-M$ or in particular for $A$, then $A-M$ is nilpotent.

Theorem 4 and its corollaries remain true if the condition of commutativity is relaxed to the restriction that all idempotents lie in the center. Without DCC however, Theorem 4 is false, for in Ex. 1, $M$ is the set of all $\sum_{1}^{n} \alpha_{i} x^{i}$, whereas $M^{\prime}$ is the set of all $\sum_{1}^{m} \beta_{j} y^{j}$ with $\sum_{1}^{m} \beta_{j}=0$. Thus $M$ and $M^{\prime}$ do not fill out all of $A$.

\section{The cases $M_{R}, M_{L}, M_{R}$ and $M_{L}$ equal to zero.}

Theorem 5. If $A$ is a ring with DCC on right ideals, then $A$ is nilpotent if and only if $M_{R}=0$ and there are no nonzero absolute left zero divisors (i.e. elements $x$ such that $x A=0$ ) in $A^{n}$ for every $n$.

Proof. In one direction the proof is clear. Assume then that $M_{R}=0$ and that there are no nonzero absolute zero divisors in $A^{n}$. By DCC we can write $A=e_{1} A+\cdots+e_{n} A+N_{0}$ where the $e_{i} A$ are indecom- 
posable right ideals and $N_{0}$ is nilpotent. If $e_{1} \neq 0$ then it is not in $M_{R}$. Then there must exist an $x^{\prime}$ in $A$ such that $e_{1} x^{\prime} \neq 0$ and such that $e_{1} x^{\prime}$ is not in $e_{1} x^{\prime} A$. Else $e_{1}$ is in $M_{R}$ by Theorem 1 . Thus $e_{1} x^{\prime} A \neq e_{1} A$. Since $e_{1} A$ is indecomposable, $e_{1} x^{\prime} A$ must be nilpotent. Since $e_{1} x^{\prime}$ $=e_{1}^{n-1} x^{\prime}$ is in $A^{n}$ for every $n, e_{1} x^{\prime} A$ cannot be zero. Let $N$ be the max. nilpotent ideal of $A$. Then $e_{1} N>e_{1} e_{1} x^{\prime} A=e_{1} x^{\prime} A \neq 0$. However the chain $e_{1} N>e_{1} N^{2}>\cdots>e_{1} N^{m}>\cdots$ terminates in zero after a finite number of steps. Then there exists an integer $w \geqq 1$ such that $e_{1} N^{w} \neq 0, e_{1} N^{w+1}=0$. Let $x^{\prime \prime}$ be an element of $N^{w}$ such that $e_{1} x^{\prime \prime} \neq 0$ and let $x=e_{1} x^{\prime \prime}$. Then $x N=0, x \neq 0$. Since $x=e_{1} x^{\prime \prime}=e_{1}^{n-1} x^{\prime \prime}$ is in $A^{n}$ for every $n, x A \neq 0$. Since $x N_{0}<x N=0$, there must exist an $e_{i}$ such that $x e_{i} A \neq 0$. However since $x N=0, x e_{i} A$ is a minimal right ideal of $A$. For if $0 \neq I \leqq x e_{2} A$, where $I$ is a right ideal of $A$, let $Q$ $=\left\{y\right.$ in $e_{i} A: x y$ is in $\left.I\right\}$. Then $Q$ is a right ideal of $A, Q \leqq e_{i} A$. If $Q \neq e_{i} A$, then $Q$ is nilpotent because $e_{i} A$ is indecomposable. Then $x Q<x N=0$ and therefore if $z$ is in $I, z=x e_{i} z^{\prime}$ for some $z^{\prime}$ in $A, e_{i} z^{\prime}$ is in $Q, x e_{i} z^{\prime}=0, I=0$. Thus $Q=e_{i} A, I=x e_{i} A$.

Finally let $y^{\prime}$ be an element of $A$ such that $x e_{i} y^{\prime} \neq 0$, and let $y=e_{i} y^{\prime}$. Then $x y$ is in $x e_{i} A, x y N=0, x y A \neq 0$ (since $x y=e_{i}^{n-2} x y$ is in $A^{n}$ for every $n$ ). Now $x y A \leqq x e_{i} A$ and since $x e_{i} A$ is minimal, $x y A=x e_{i} A$. Therefore $x y$ is in $x y A$. Furthermore if $x y u \neq 0, x y u A \neq 0$ (again since $x y u=e_{i}^{n-3} x y u$ is in $A^{n}$ for every $n$ ) and thus $x y u$ is in $x y u A$. Therefore $x y$ is in $M_{R}$ by Theorem 1 . This is a contradiction and thus all the $e_{i}$ are zero, and $A$ must be nilpotent. Q.E.D.

Corollary 1. If $A$ has DCC on left ideals then $A$ is nilpotent if and only if $M_{L}=0$ and there are no nonzcro absolute right zerodivisors in $A^{n}$ for coery $n$.

Since $M_{R}$ contains the max. regular ideal $\bar{M}$ and when $A-J$ is regular, or in particular when $A$ has DCC on right ideals, then $\bar{M}=0$ if and only if $A$ is bound to $J$, Theorem 6, [4], we can conclude that when $M_{R}=0$ and $A$ has DCC on right ideals, $A$ is bound to $J$. Combining this with Theorem 5 we have

Corollary 2. If $A$ has $D C C$ on right ideals and $M_{R}=0$, then either $A$ is nilpotent or $A$ is bound to $N$ and $A$ has an absolute left zero divisor in $A^{n}$ for every $n$.

The converse is also true. Using the fact that $M_{R}\left(A-M_{R}\right)=0$ we have

Corollary 3. If $A$ has $D C C$ on right ideals, then $A-M_{R}$ is nilpotent if and only if $x A \leqq M_{R}$ and $x$ in $\left(A-M_{R}\right)^{n}$ for every $n$, implies that $x$ is in $M_{R}$. 
When $M_{R}=M_{L}=0$ the zero divisor condition can be slightly weakened.

Theorem 6. If $A$ has DCC on one-sided ideals, then $A$ is nilpotent if and only if $M_{R}=M_{L}=0$ and there are no nonzero total divisors of zero in $A^{n}$ for every $n$, i.e. elements $x$ such that $x A=A x=0$ and $x$ in $A^{n}$ for every $n$.

Proof. In one direction the proof is clear. Conversely, we write as before $A=e_{1} A+\cdots+e_{n} A+N_{0}$ where the $e_{i} A$ are indecomposable right ideals and $N_{0}$ is nilpotent. If $e_{1} \neq 0$, consider the chain $e_{1} N$ $>e_{1} N^{2}>\cdots>e_{1} N^{\alpha}=0$. There exists an integer $\gamma$ such that $e_{1} N^{\gamma} \neq 0$, $e_{1} N^{\gamma+1}=0$. If $\gamma=0$, let $x$ be any element such that $e_{1} x \neq 0$. Consider $e_{1} x A \leqq e_{1} A$. If $e_{1} x A \neq e_{1} A$, then since $e_{1} A$ is indecomposable, $e_{1} x A$ is nilpotent, $e_{1} x A$ is in $N$. Then $e_{1} \cdot e_{1} x A<e_{1} N=0$. Then $e_{1} x$ is properly nilpotent, $e_{1} x$ is in $N$. Then $e_{1} \cdot e_{1} x=0=e_{1} x$, a contradiction. On the other hand if $e_{1} x A=e_{1} A$ then $e_{1} x$ is in $e_{1} x A$ and since $e_{1}$ is in $e_{1} A, e_{1}$ is in $M_{R}$ by Theorem 1 . Then $e_{1}=0$, a contradiction. Thus $e_{1} N \neq 0, \gamma \geqq 1$. Similarly there exists an integer $\rho \geqq 1$ such that $N^{\rho} e_{1} \neq 0, N^{\rho+1} e_{1}=0$. In this way we obtain for each $e_{i}$, integers $\gamma_{i}$ and $\rho_{i}$ such that $e_{i} N^{\gamma_{i}} \neq 0$, $e_{i} N^{\gamma_{i}+1}=0, N^{\rho_{i}} e_{i} \neq 0, N^{\rho_{i}+1} e_{i}=0$. Let $\beta$ be the maximum of the $\gamma_{i}$ and $\rho_{i}$. Then setting $e=e_{1}+\cdots+e_{n}, e N^{\beta+1}=N^{\beta+1} e=0$, and either $e N^{\beta}$ or $N^{\beta} e \neq 0$. Suppose $e N^{\beta} \neq 0$. Then for some $e_{j}, e_{j} N^{\beta} \neq 0$. Take $x^{\prime}$ in $N^{\beta}$ such that $e_{j} x^{\prime} \neq 0$ and let $x=e_{j} x^{\prime}=e_{j} x=e x$. Then $x N=0$. Since $M_{L}=0$ and $x$ is in $A x$, there must exist (Theorem 1) an element $y$ in $A$ such that $y x \neq 0$ and such that $y x$ is not in $A y x$. We may take $y=y e_{j}=y e$. The element $y$ must be in $N$. For $y x$ not in $A y x$ implies $y$ not in $A y=A y e_{j}$. Thus $A y e_{j} \neq A e_{j}$ and since $A e_{j}$ is indecomposable, $A y e_{j}$ is nilpotent. Then $y e_{j} \cdot y e_{j}$ is nilpotent, $y e_{j}$ is nilpotent and clearly $y e_{j}=y$ is properly nilpotent and therefore $y$ is in $N$. Then $y x$ is in $N e_{j} N^{\beta} \leqq N^{\beta+1}$ and therefore $y x e=0$. Also $y x N=0$ and thus $y x A$ $=0$. Also $e y x=0$, since $e N^{\beta+1}=0$. Note that $y x=y e_{j} x=y e_{j}^{n-2} x$ is in $A^{n}$ for every $n$. If $y x$ is not a total zerodivisor, $A y x \neq 0$. Then, $N y x \neq 0$, since $e y x=0$. Let $y_{1}$ be an element in $N$ such that $y_{1} y x \neq 0$. As above $y_{1} y x A=e y_{1} y x=0$. If $y_{1} y x$ is not a total divisor of zero, $N y_{1} y x \neq 0$. We continue this process until $t=y_{\beta-1} \cdots y_{1} y x \neq 0, t A=0$, et $=0$. Then $t$ is in $N^{\beta} e_{j} N^{\beta}$ and $N t$ is in $N^{\beta+1} e_{j} N^{\beta}=0$. Thus $A t=t A=0$, and $t$ $=y_{\beta-1} \cdots y_{1} y e_{j}^{m} x$ is in $A^{n}$ for every $n$. This is impossible and thus $e_{i}=0$ for every $i, A=N_{0}, A$ is nilpotent. Q.E.D.

The Hopkins example mentioned earlier shows that the divisor of zero restrictions cannot be removed, for $M_{R}=0, A$ has DCC and is not nilpotent. To obtain an example for Theorem 6 , let $A$ be an algebra of dimension 4 over a field $F$, with basal elements $e, u, v, w$ and the following multiplication table: 


\begin{tabular}{c|cccc} 
& $e$ & $u$ & $v$ & $w$ \\
\hline$e$ & $e$ & 0 & $v$ & 0 \\
$u$ & $u$ & 0 & $w$ & 0 \\
$v$ & 0 & 0 & 0 & 0 \\
$w$ & 0 & 0 & 0 & 0
\end{tabular}

Then $M_{R}=M_{L}=0$ and $A$ is not nilpotent. The radical is generated by $u, v$, and $w$. The element $w$ is a total divisor of zero and in $A^{n}$ for every $n$. This algebra is in fact subdirectly irreducible with minimal ideal generated by $w$. For let $I$ be any ideal of $A$, with $x=\alpha e+\beta u+\gamma v$ $+\delta w$ in $I$. Then $x e=\alpha e+\beta u, e x=\alpha e+\gamma v, x v=\alpha v, u x=\alpha u$. Then if $\alpha \neq 0, I$ contains $u, v$ and then $w$, and also $e, I=A$. If $\alpha=0, I$ contains $\beta u$ and $\gamma v$ and therefore $\delta w$. If $\beta \neq 0$, then $u$ and $w$ are in $I$. If $\gamma \neq 0$, $v$ and $w$ are in $I$. If $\beta=\gamma=0, I=(w)$. Thus there are precisely five nonzero ideals: $(w),(u, v),(v, w),(u, v, w),(u, v, w, e)$. Since $w$ is not in $M_{R}$ or $M_{L}$ they are zero.

We now return to the commutative case but drop DCC. Then $M=\{x: x$ is in $x A\}$ and thus $M$ contains all idempotents. In (1) all $M_{i}^{\prime}=$ the corresponding $M_{i}$.

Theorem 7. If $A$ is commutative then $M=0$ if and only if $A$ is isomorphic to a subdirect sum of subdirectly irreducible rings with an absolute divisor of zero in their minimal ideals. That is, they are of type $\beta$ $[7]$.

From [7] we know that a commutative subdirectly irreducible ring with the ascending chain condition is either nilpotent or has a unity element. Thus we have

Theorem 8. If $A$ is commutative with $A C C$ then $M=0$ if and only if $A$ is isomorphic to a subdirect sum of nilpotent subdirectly irreducible rings.

Though Theorems 7 and 8 seem to yield radical-like results, this may be misleading. Let $A$ be the ring of even integers. It has ACC but not DCC. Also $M=0$ and $A$ is isomorphic to a subdirect sum of nilpotent rings, namely

$$
A=\left(A /(4), A /(8), \cdots, A /\left(2^{n}\right), \cdots\right)
$$

where $A /\left(2^{n}\right)$ has $2^{n-1}$ as an absolute divisor of zero and is nilpotent for every $n$. However $J=0$ and therefore $A$ is isomorphic to a subdirect sum of fields, namely

$$
A=(A /(6), A /(10), A /(14), \cdots, A /(2 p), \cdots)
$$


where $p$ is a prime. Thus $A$ may be semi-simple and still have $M=0$.

This example also shows that DCC is necessary to obtain nilpotence. To see that ACC is necessary to obtain a subdirect sum of nilpotent rings, we may consider the example in [7] which is commutative, subdirectly irreducible, has neither chain condition, has $M=0$ and is not nilpotent.

3. The cases $A=M_{R}, A=M_{L}, A=M_{R}=M_{L}$. In studying the existence of right, left and two sided unities, Baer $[1 ; 2 ; 3]$, concerned himself to some extent with right and left $D$-regularity. We summarize some of his results in the language of $M_{R}, M_{L}$ and $M$ :

With DCC on one-sided ideals:

1a. $A=M_{R}$ if and only if $A$ has a right unity.

2a. $A=M_{R}=M_{L}$ if and only if $A$ has a unity.

3a. $A$ commutative, $A=M$ if and only if $A$ has a unity.

If $A-J$ has a unity or if $A-J$ has DCC on one-sided ideals:

2b. $A=M_{R}=M_{L}$ if and only if $A$ has a unity.

3b. $A$ commutative, $A=M$ if and only if $A$ has a unity.

However 1 a needed some strengthening:

1b. $A$ has a right unity if and only if $A=M_{R}$ and when $A=J+A x$, $x$ must be in $A x$.

Baer also proved, for a ring with DCC on one-sided ideals:

c. $A$ has a right unity if and only if $A$ has a non-right-zero divisor, i.e. an element $x$ such that $y x=0$ implies $y=0$.

Let $A$ be a ring with ACC on left ideals. Then, as is well known, every left ideal of $A$ is finitely generated and in particular $M_{R}$ $=\left\{\sum_{1}^{m} n_{i} a_{i}+x_{i} a_{i}\right\}, x_{i}$ in $A, a_{i}$ in $M_{R}, n_{i}$ integers. By the corollary to Lemma 5, there exists an element $e$ in $A$ such that $M_{R}=M_{R} e$, pointwise. If we assume $M_{R}=A, e$ is a right unity element.

Theorem 9. If $A$ has $A C C$ on left ideals, then $A$ has a right unity if and only if $A=M_{R}$.

Passing now to rings without chain conditions, we first prove

Lemma 6. $A$ has a left unity if and only if there exists an element $x$ in $A$ such that $x$ is in $x A$ and $x$ is not a left zero divisor.

Proof. If $A$ has a left unity $f$, then $f$ is in $f A$, and if $f y=0$ then clearly $y=0$. Conversely if $x$ is in $x A, x=x e$, then for every $y, x(y-e y)$ $=0$ and since $x$ is not a left zero divisor, $y=e y, e$ is a left unity.

Note that if $x$ were also not a right zero divisor, then $A$ would have a unity. For $x=e x=x e$ and $(y-y e) x=0$ yields $y=y e$ for every $y$.

Corollary. A has a unity if and only if either $M_{R}$ or $M_{L}$ has a non-zero-divisor. 
We thus have for rings without chain conditions:

THEOREM 10.

a. If $A=M_{R}$, then $A$ has a left unity if and only if $A$ has a non-leftzero-divisor; $A$ has a unity if and only if $A$ has a non-zero-divisor.

b. If $A=M_{L}, A$ has a right unity if and only if $A$ has a non-rightzero-divisor.

c. If $A=M_{R}=M_{L}$, then $A$ has a unity if and only if $A$ has a nonzero-divisor if and only if $A$ has a non-right and a non-left-zero-divisor.

Note that then if $A=M_{R}=M_{L}$ and if $A$ has neither a right nor a left unity, then every element of $A$ is a two-sided divisor of zero.

In the commutative case, when $A=M$ it is thus clear that $A$ has a unity if and only if $A$ has a non-zero-divisor. We can obtain more. For when $A$ is expressed as a subdirect sum of subdirectly irreducible rings $A_{i}$, each $A_{i}$ must be equal to its $M_{i}$ : Let $x_{i}$ be any element of $A_{i}$. It must appear in the expansion of some element, say $x=\left(x_{1}, \cdots\right.$, $\left.x_{i}, \cdots\right)$. Since $A=M$, there exists an element $y$ in $A$ such that $x y=x$. Let $y=\left(y_{1}, \cdots, y_{i}, \cdots\right)$. Then $x_{i} y_{i}=x_{i}$ and $x_{i}$ is in $M_{i}$, $A_{i}=M_{i}$. However from [7], it is clear that if a commutative subdirectly irreducible ring is equal to its maximal $D$-regular ideal, it has a unity element. In fact it is either a field or has a unity and is a field modulo its set (an ideal) of zero-divisors. If in addition it has ACC, it is either a field or has a unity and is a field modulo its maximal nilideal. Thus we have:

Theorem 11. If $A$ is a commutative ring and $A=M$, then $A$ is isomorphic to a subdirect sum of subdirectly irreducible rings each with a unity. Some are fields and others are fields modulo the ideal of zerodivisors. If $A$ has $A C C$, the latter set are ficlds modulo their maximal nilideals.

Of course $A$ itself may not have a unity, for let $A$ be the weak direct sum of an infinite number of fields. Every element of $A$ is a zero-divisor and $A$ has no unity.

In summary we have:

$A=M_{R}=M_{L}$ if and only if $A$ has a unity and any one of the five following conditions: DCC on one-sided ideals; ACC on one-sided ideals; a unity element in $A-J$; a non-zero-divisor; a non-left and non-right-zero-divisor.

$A=M_{R}$ if and only if $A$ has a right unity and one of the following three conditions: DCC on right ideals; ACC on left ideals; $A-J$ has a unity element and $A=J+A x$ implies that $x$ must be in $A x$. 
$A=M_{R}$ implies that $A$ has a left unity, if it has a non-left-zerodivisor.

$A=M_{R}$ if and only if $A$ has a unity, if $A$ has a non-zero-divisor.

\section{BibLIOGRAPHY}

1. Baer, Inverses and zero divisors, Bull. Amer. Math. Soc. vol. 48 (1942) pp. 630638.

2. —_ Radical ideals, Amer. J. Math. vol. 65 (1943) pp. 537-568.

3. - Kriterien fur die Existence eines Einzelelement in Ringen, Math. Zeit. vol. 56 (1952) pp. 1-17.

4. Brown and McCoy, The maximal regular ideal of a ring, Proc. Amer. Math. Soc. vol. 1 (1950) pp. 165-171.

5. - Some theorems on groups, Trans. Amer. Math. Soc. vol. 69 (1950) pp. 302-311.

6. Divinsky, Pseudo regularity, Canadian J. Math. vol. 7 (1955) pp. 401-410.

7. - Commutative subdirectly irreducible rings, Proc. Amer. Math. Soc. vol. 8 (1957) pp. 642-648.

8. Hopkins, Rings with minimal condition for left ideals, Ann. of Math. vol. 40 (1939) pp. 712-730.

9. McCoy, Commutative subdirectly irreducible rings, Duke Math. J. vol. 12 (1945) pp. 381-387.

UNIVERSity OF MANITOBA 